\title{
A FINITE-DIMENSIONAL APPROACH TO WAVELET SYSTEMS ON THE CIRCLE
}

\author{
Brody Dylan Johnson
}

Saint Louis University, USA

\begin{abstract}
Motivated by recent developments in the study of finitedimensional frames, this work develops an independent theory of finitedimensional wavelet systems on the circle. Using natural translation and dilation operators, trigonometric polynomial, orthonormal scaling functions are constructed which give rise to finite-dimensional multiresolution analyses and, consequently, orthonormal wavelet systems. It is shown that the finite-dimensional systems so constructed can lead to arbitrarily close approximation of square-integrable functions on the circle. Departures from the existing theory of periodic wavelets are encountered, e.g., the finite-dimensional equivalent of the Smith-Barnwell equation describes both a necessary and sufficient condition on a candidate low-pass filter for the existence of an orthonormal scaling function. Moreover, this finite-dimensional framework allows for a natural analog to the Shannon wavelet, in contrast to the classical periodic wavelets.
\end{abstract}

\section{INTRODUCTION}

Wavelets on the circle, or periodic wavelets, have been studied by a number of previous authors. The earliest constructions (see [8] or [5]) rely on the periodization of functions on the line through application of the operator $P: L^{1}(\mathbb{R}) \rightarrow L^{1}(\mathbb{T})$

$$
\operatorname{Pf}(x)=\sum_{k \in \mathbb{Z}} f(x+k), \quad x \in \mathbb{T},
$$

where $\mathbb{T}$ will be associated with the interval $[0,1)$. In such constructions, periodization of an $L^{1}$-scaling function associated with a cardinal multiresolution analysis leads to a multiresolution structure and associated wavelet

2010 Mathematics Subject Classification. 42C15, 65T60.

Key words and phrases. Wavelet, circle, periodic wavelet, multiresolution analysis. 
basis for $L^{2}(\mathbb{T})$. Another approach to periodic wavelets involves the direct construction of multiresolution structures on the circle independent of the theory on the line, e.g., [3], [9], and [2]. Each of these constructions bears elements of the usual wavelet theory on the line, yet none explicitly considers a notion of dilation on the circle. Moreover, each is concerned with the study of infinite-dimensional systems in $L^{2}(\mathbb{T})$.

The goal of this article is to develop a notion of finite-dimensional wavelet theory for the circle which is rooted in its inherent algebraic structure. A natural choice for translation is given by the finite group action of $\mathbb{Z}_{N}=\mathbb{Z} / N \mathbb{Z}$ $(N \in \mathbb{N})$ via the circular translation,

$$
T_{N} f(x)=f\left(x-N^{-1}\right), \quad x \in \mathbb{T} .
$$

The space of the $N$-dimensional space of complex-valued functions on $\mathbb{Z}_{N}$ will be denoted by $\ell\left(\mathbb{Z}_{N}\right)$. Dilation will be achieved by the mapping $D: L^{2}(\mathbb{T}) \rightarrow$ $L^{2}(\mathbb{T})$ defined by

$$
D f(x)=2^{-1}\left(f\left(2^{-1} x\right)+f\left(2^{-1} x+2^{-1}\right)\right), \quad x \in \mathbb{T} .
$$

This definition is motivated in part by the relationship between dilation on the line and the periodization map $P$ discussed above, but also by the commutativity relationship this creates for $T_{N}$ and $D$. Before explaining this further, it is convenient to pause long enough to establish notation for Fourier analysis on $\mathbb{T}, \mathbb{Z}$, and $\mathbb{Z}_{N}$.

1. The Fourier transform of $f \in L^{2}(\mathbb{T})$ will be denoted by $\hat{f}$ and is given by

$$
\hat{f}(k)=\int_{\mathbb{T}} f(x) e^{-2 \pi i k x} d x, \quad k \in \mathbb{Z} .
$$

These are the Fourier series coefficients of $f$.

2. The Fourier transform of $f \in \ell^{2}(\mathbb{Z})$ will also be denoted by $\hat{f}$ and is defined for $f \in \ell^{1} \cap \ell^{2}(\mathbb{Z})$ by

$$
\hat{f}(\xi)=\sum_{k \in \mathbb{Z}} f(k) e^{-2 \pi i k \xi}, \quad \xi \in \mathbb{T} .
$$

3. The Fourier transform on $\ell\left(\mathbb{Z}_{N}\right)$ will be denoted by $\mathcal{F}_{N}: \ell\left(\mathbb{Z}_{N}\right) \rightarrow$ $\ell\left(\mathbb{Z}_{N}\right)$ and is defined for $f \in \ell\left(\mathbb{Z}_{N}\right)$ by

$$
\mathcal{F}_{N} f(n)=\hat{f}(n)=\frac{1}{\sqrt{N}} \sum_{k \in \mathbb{Z}_{N}} f(k) e^{-2 \pi i k n / N}, \quad n \in \mathbb{Z}_{N} .
$$

The meaning of the hat-notation for the Fourier transform will be clear from context. Now, to examine the relationship between $D$ and $T_{N}$, observe that for $f \in L^{2}(\mathbb{T})$,

$$
\widehat{D f}(k)=\hat{f}(2 k) \quad \text { and } \quad \widehat{T_{N} f}(k)=e^{-2 \pi i k / N} \hat{f}(k), \quad k \in \mathbb{Z} .
$$

It follows from these identities that $T_{N}^{2} D=D T_{N}$, which mirrors the relationship between translation and dilation on $\mathbb{R}$ and plays an important 
role in the development of multiresolution structures (see [6]). Also note that the above identity shows that $D$ performs a downsampling of the Fourier coefficients of functions in $L^{2}(\mathbb{T})$ and, therefore, is not invertible. One implication of this observation is that any associated multiresolution structure on the circle must be one-sided, i.e., only positive powers of the dilation operator may be used.

The organization of the article is as follows. Section 2 describes basic facts about principal shift-invariant subspaces of $L^{2}(\mathbb{T})$ in terms of a finite-dimensional bracket product. In particular, the bracket product provides a simple characterization of functions whose translates over $\mathbb{Z}_{N}$ are orthonormal. Section 3 consists of a study of refinable functions on the circle which leads to a corresponding notion of finite-dimensional multiresolution analyses (MRAs). The main results of this work are presented in this section. Theorem 3.5 provides a characterization of those refinable functions which are orthonormal scaling functions for an MRA in terms of the bracket product and a finite-dimensional version of the familiar Smith-Barnwell equation. Meanwhile, Theorem 3.6 shows that given a candidate low-pass filter satisfying the Smith-Barnwell equation, one can construct a corresponding orthonormal scaling function of any finite order $N=2^{J}$. The lack of further restrictions on the low-pass filter, such as Cohen's condition, illustrates the added flexibility found in the finite-dimensional setting and represents a significant departure from MRA theory on the real line. Section 4 builds on the MRA theory developed in Section 3 by offering a corresponding description of orthonormal wavelet bases for certain subspaces of $L^{2}(\mathbb{T})$. Finally, in Section 5 , the approximation error of the systems constructed in Sections 3 and 4 is considered. It is shown that the finite-dimensional systems developed in Sections 3 and 4 can lead to arbitrarily small approximation error for functions in $L^{2}(\mathbb{T})$. The section concludes with an example motivated by the Shannon wavelet on $\mathbb{R}$, which is not suitable for the periodization approach found in the classical treatments of periodic wavelets.

\section{PRINCIPAL SHIFT-INVARIANT SPACES}

The theory of shift-invariant spaces on the line is well understood in terms of the bracket product studied by de Boor, DeVore, and Ron ([1]) and later by Ron and Shen ([10]). The machinery developed on the real line carries over quite naturally to the circle as the translation group $\mathbb{Z}$ found on $\mathbb{R}$ is replaced by $\mathbb{Z}_{N}$ on $\mathbb{T}$. Rather than develop this theory in its entirety, however, the purpose of this section is to collect only the results necessary in the subsequent sections. The reader is referred to the work of Plonka and Tasche ([9]) for a broader treatment of this material which deals with multiple generators and the case of general frames. Basic information about frames can be found in the texts [4], [5], and [7]. 
Definition 2.1. The bracket product of order $N$ of two functions $f, g \in$ $L^{2}(\mathbb{T})$ is the vector $[\hat{f}, \hat{g}]_{N} \in \ell\left(\mathbb{Z}_{N}\right)$ defined by

$$
[\hat{f}, \hat{g}]_{N}(n)=N \sum_{k \in \mathbb{Z}} \hat{f}(n+k N) \overline{\hat{g}(n+k N)}, \quad 0 \leq n \leq N-1 .
$$

Notice that the square-integrability of $f$ and $g$ ensures that $[\hat{f}, \hat{g}]$ is welldefined. As on the line, the bracket product of $f$ and $g$ on the circle is closely related to the inner products of $f$ with the translates of $g$. In particular, the following proposition shows how the finite-dimensional Fourier transform relates the two objects.

Proposition 2.2. For all $f, g \in L^{2}(\mathbb{T})$,

$$
\mathcal{F}_{\mathcal{N}}\left(\left\{\left\langle f, T_{N}^{n} g\right\rangle\right\}_{n=0}^{N-1}\right)=\frac{1}{\sqrt{N}}[\hat{f}, \hat{g}]_{N} .
$$

Proof. For $0 \leq n \leq N-1$ observe that

$$
\begin{aligned}
\left\langle f, T_{N}^{n} g\right\rangle & =\sum_{k \in \mathbb{Z}} \hat{f}(k) \overline{\widehat{T_{N}^{n}} g(k)}=\sum_{k \in \mathbb{Z}} \sum_{\ell=0}^{N-1} \hat{f}(\ell+k N) \overline{\hat{g}(\ell+k N)} e^{2 \pi i n \ell / N} \\
& =\frac{1}{N} \sum_{\ell=0}^{N-1}[\hat{f}, \hat{g}]_{N}(\ell) e^{2 \pi i n \ell / N}=\frac{1}{\sqrt{N}}\left(\mathcal{F}_{N}^{-1}[\hat{f}, \hat{g}]_{N}\right)(n) .
\end{aligned}
$$

The following definition makes clear how the finite groups $\mathbb{Z}_{N}$ lead to shift-invariant spaces on the circle.

Definition 2.3. Given $\phi \in L^{2}(\mathbb{T})$ and $N \in \mathbb{N}$, the principal shiftinvariant space of order $N$ generated by $\phi$ is the finite-dimensional space $V_{N}(\phi)$ given by

$$
V_{N}(\phi)=\operatorname{span}\left\{T_{N}^{n} \phi: 0 \leq n \leq N-1\right\} .
$$

The collection $\left\{T^{n} \phi: 0 \leq n \leq N-1\right\}$ will be denoted hereafter by $X_{N}(\phi)$.

The following characterization of orthonormal generators of PSI spaces is an immediate consequence of Proposition 2.2.

Corollary 2.4. Let $\phi \in L^{2}(\mathbb{T})$. Then $X_{N}(\phi)$ is an orthonormal basis for $V_{N}(\phi)$ if and only if $[\hat{\phi}, \hat{\phi}]_{N}(n)=1, n \in \mathbb{Z}_{N}$.

\section{RefinABle FUnCtions AND MUltiRESOlUtion ANALYSiS}

In this section, a notion of multiresolution analysis will be developed through an examination of refinable functions on the circle. 
Definition 3.1. A function $\phi \in L^{2}(\mathbb{T})$ is said to be refinable of order $N$ $(N \in \mathbb{N})$ if there exists a mask $c \in \ell\left(\mathbb{Z}_{N}\right)$ such that

$$
D \phi=\sum_{n \in \mathbb{Z}_{N}} c(n) T_{N}^{n} \phi .
$$

As on the line, the refinability of a function on the circle is well understood in terms of its Fourier transform.

Lemma 3.2. Suppose that $\phi \in L^{2}(\mathbb{T})$ is refinable of order $N$, then there exists $m \in \ell\left(\mathbb{Z}_{N}\right)$ such that

$$
\hat{\phi}(2 k)=m(k) \hat{\phi}(k), \quad k \in \mathbb{Z} .
$$

Proof. By hypothesis, there exists $c \in \ell\left(\mathbb{Z}_{N}\right)$ such that (3.1) holds, which is equivalent to

$$
\hat{\phi}(2 k)=\left(\sum_{n \in \mathbb{Z}_{N}} c(n) e^{-2 \pi i n k / N}\right) \hat{\phi}(k), \quad k \in \mathbb{Z} .
$$

Thus, defining $m(k)=\sqrt{N} \hat{c}(k), k \in \mathbb{Z}_{N}$, the lemma is proven.

The finite-dimensional vector $m$ in Lemma 3.2 is often referred to as the filter associated with the refinable function $\phi$. Observe that if $\phi$ is a refinable function, then the lemma implies that either $\hat{\phi}(0)=0$ or the associated filter $m$ satisfies $m(0)=1$. Thus, it is necessary that $m(0)=1$ if the associated refinable function is to have a nonzero integral.

Definition 3.3. A multiresolution analysis (MRA) of order $N=2^{J}$ $(J \in \mathbb{N})$ is a collection of closed subspaces of $L^{2}(\mathbb{T}),\left\{V_{j}\right\}_{j=0}^{J}$, satisfying

1. For $0 \leq j \leq J-1, V_{j+1} \subseteq V_{j}$;

2. For $0 \leq j \leq J-1, f \in V_{j}$ if and only if $D f \in V_{j+1}$;

3. $V_{J}$ is the subspace of constant functions;

4. There exists a scaling function $\varphi \in V_{0}$ such that $X_{2^{-j} N}\left(2^{\frac{j}{2}} D^{j} \varphi\right)$ is an orthonormal basis for $V_{j}$.

Notice that MRA properties (1.) and (4.) imply that a scaling function $\varphi$ is necessarily refinable of order $N$. Moreover, it follows from MRA properties (3.) and (4.) that $D^{J} \varphi$ must be constant and nonzero, implying that $\hat{\varphi}(0) \neq 0$.

REMARK 3.4. Suppose that $\varphi \in L^{2}(\mathbb{T})$ is refinable of order $N=2^{J}$ $(J \in \mathbb{N})$ with associated filter $m \in \ell\left(\mathbb{Z}_{N}\right)$. Applying the dilation operator to (3.1) $j$ times, one obtains

$$
D^{j+1} \varphi=\sum_{n \in \mathbb{Z}_{N}} c(n) D^{j} T_{N}^{n} \varphi .
$$


Recall that $T_{N}^{2} D=D T_{N}$, so $D^{j} T_{N}^{n}=T_{N}^{2^{j} n} D^{j}=T_{2^{-j} N}^{n} D^{j}$, so the above refinement equation can be restated as

$$
D^{j+1} \varphi=\sum_{n \in \mathbb{Z}_{N}} c(n) T_{2^{-j} N}^{n} D^{j} \varphi
$$

Now, observe that the summation over $\mathbb{Z}_{N}$ results in duplicated translates, allowing for another restatement with the sum over $\mathbb{Z}_{2^{-j} N}$,

$$
D^{j+1} \varphi=\sum_{n \in \mathbb{Z}_{2-j}}\left(\sum_{\ell=0}^{2^{j}-1} c\left(n+\ell 2^{-j} N\right)\right) T_{2^{-j} N}^{n} D^{j} \varphi .
$$

The upshot of these observations is that $D^{j} \varphi$ is refinable of order $2^{-j} N$ and the associated mask is obtainable from the original via periodization. Denoting the mask as $c_{j} \in \ell\left(\mathbb{Z}_{2^{-j} N}\right)$, observe that the corresponding filter is $\left(2^{-j} N\right)^{\frac{1}{2}} \widehat{c_{j}}=m\left(2^{j} \cdot\right)$.

The next theorem characterizes those refinable functions which are scaling functions for an MRA in terms of the bracket product and a variation of the familiar Smith-Barnwell equation for low-pass filters.

THEOREM 3.5. Suppose $\varphi \in L^{2}(\mathbb{T})$ is a refinable function of order $N=2^{J}$ $(J \in \mathbb{N})$ with $\hat{\varphi}(0) \neq 0$. Then $\varphi$ is the scaling function of an MRA of order $N$ if and only if

$$
\left|m_{0}(k)\right|^{2}+\left|m_{0}\left(k+2^{-1} N\right)\right|^{2}=1, \quad k \in \mathbb{Z}_{N},
$$

$$
[\hat{\varphi}, \hat{\varphi}]_{N}(n)=1, \quad n \in \mathbb{Z}_{N} .
$$

Proof. Some preliminary observations will simplify the proof. Recall that the dilation operator is not unitary, but satisfies $T_{N}^{2} D=D T_{N}$. Hence, if $\varphi$ is refinable (satisfies (3.1)) then

$$
T_{2^{-1} N}^{k} D \varphi=T_{N}^{2 k} D \varphi=\sum_{n \in \mathbb{Z}_{N}} c(n) T_{N}^{n+2 k} \varphi, \quad 0 \leq k \leq 2^{J-1}-1 .
$$

Moreover, observe that

$$
\begin{aligned}
{\left[2^{\left.\frac{1}{2} \widehat{D \varphi}, 2^{\frac{1}{2}} \widehat{D \varphi}\right]_{\frac{N}{2}}(n)=}\right.} & N \sum_{k \in \mathbb{Z}}\left|\widehat{D \varphi}\left(n+k \frac{N}{2}\right)\right|^{2} \\
= & N \sum_{k \in \mathbb{Z}}\left|\hat{\varphi}\left(n+k \frac{N}{2}\right)\right|^{2}\left|m_{0}\left(n+k \frac{N}{2}\right)\right|^{2} \\
= & N\left[\sum_{k \in \mathbb{Z}}|\hat{\varphi}(n+k N)|^{2}\left|m_{0}(n+k N)\right|^{2}\right. \\
& \left.+\sum_{k \in \mathbb{Z}}\left|\hat{\varphi}\left(n+\frac{N}{2}+k N\right)\right|^{2}\left|m_{0}\left(n+\frac{N}{2}+k N\right)\right|^{2}\right]
\end{aligned}
$$




$$
=[\hat{\varphi}, \hat{\varphi}]_{N}(n)\left|m_{0}(n)\right|^{2}+[\hat{\varphi}, \hat{\varphi}]_{N}\left(n+\frac{N}{2}\right)\left|m_{0}\left(n+\frac{N}{2}\right)\right|^{2}
$$

The necessity of (3.4) follows from the $j=0$ statement of MRA property 4. and Theorem 2.4. Given (3.4), the above calculation shows that

$$
\left[2^{\frac{1}{2}} \widehat{D \varphi}, 2^{\frac{1}{2}} \widehat{D \varphi}\right]_{2^{-1} N}(n)=\left|m_{0}(n)\right|^{2}+\left|m_{0}\left(n+2^{-1} N\right)\right|^{2}
$$

demonstrating the necessity of (3.3).

To see the sufficiency, assume that $m_{0}$ satisfies (3.3) and $\varphi$ satisfies (3.4). Define $V_{j}=V_{2^{-j} N}\left(2^{\frac{j}{2}} D^{j} \varphi\right), 0 \leq j \leq J$. MRA property (2.) is immediate, while MRA property (1.) follows from the refinability of $\varphi$ in light of the preliminary observations above.

It is assumed that $\hat{\varphi}(0) \neq 0$, so Lemma 3.2 implies that $m_{0}(0)=1$ and (3.3) implies $m_{0}\left(2^{J-1}\right)=0$. Observe that

$$
\widehat{D^{J} \varphi}(k)=\hat{\varphi}\left(2^{J} k\right)=\hat{\varphi}(k) \prod_{j=0}^{J-1} m_{0}\left(2^{j} k\right), \quad k \in \mathbb{Z} .
$$

If $k \notin N \mathbb{Z}$, then the product on the right-hand side of the last equality must contain a factor equal to $m_{0}\left(2^{J-1}\right)$, which is zero. For $k \in N \mathbb{Z}$ notice that $\hat{\varphi}(k)$ can be expanded similarly,

$$
\hat{\varphi}(k)=\hat{\varphi}\left(2^{-J} k\right) \prod_{j=0}^{J-1} m_{0}\left(2^{j-J} k\right),
$$

so $\hat{\varphi}(k)=0$ by the same reasoning. Therefore $\widehat{D^{J} \varphi}(k)=0$ for all $k \neq 0$, while $\widehat{D^{J} \varphi}(0)=\hat{\varphi}(0) \neq 0$. This proves that $D^{J} \varphi$ is a constant, nonzero function and demonstrates MRA property (3.).

Finally, observe that the calculation of $\left[2^{\frac{1}{2} \widehat{D \varphi}}, 2^{\frac{1}{2} \widehat{D \varphi}}\right]_{2^{-1} N}$ given above shows that $X_{2^{-1} N}\left(2^{\frac{1}{2}} D \varphi\right)$ is an orthonormal basis for $V_{1}$. The corresponding statement for $V_{j}, 2 \leq j \leq J$, follows by induction in light of Remark 3.4, completing the demonstration of MRA property (4.) as well as the proof of the theorem.

The following theorem shows that a candidate low-pass filter $m_{0}$ satisfying $m_{0}(0)=1$ and (3.3) always leads to a corresponding trigonometric polynomial scaling function. This is somewhat surprising given that the Smith-Barnwell condition is far from sufficient for the existence of an orthonormal scaling function on the real line, yet, at the same time, it is the leap from finite to infinite which prompts further restrictions on the filter, e.g., Cohen's condition $([5])$.

TheOREM 3.6. Fix $N=2^{J}, J \in \mathbb{N}$. Suppose $m_{0} \in \ell\left(\mathbb{Z}_{N}\right)$ satisfies (3.3) with $m_{0}(0)=1$. Then $m_{0}$ is the low-pass filter of a trigonometric polynomial scaling function of order $N$. 
PROOF. The proof will consist of the construction of an orthonormal scaling function $\varphi$ associated with $m_{0}$. Combining Lemma 3.2 with Theorem 2.4 , respectively, $\varphi$ must satisfy

$$
\hat{\varphi}(2 k)=m_{0}(k) \hat{\varphi}(k), \quad k \in \mathbb{Z},
$$

and

$$
1=N \sum_{k \in \mathbb{Z}}|\hat{\varphi}(n+k N)|^{2}, \quad n \in \mathbb{Z}_{N}
$$

Notice that (3.5) can be used to specify $\hat{\varphi}$ recursively on dyadic sequences originating at odd integers. In order to satisfy (3.6) for each odd index $n \in \mathbb{Z}_{N}$ it is sufficient to specify $\hat{\phi}(k)$ for each odd index between $-2^{-1} N$ and $2^{-1} N$. With these preliminary observations $\hat{\varphi}$ will be constructed as follows.

1. Let $\hat{\varphi}(0)=\frac{1}{\sqrt{N}}$.

2. For $-2^{J-2} \leq k \leq 2^{J-2}-1$, let $\hat{\varphi}(2 k+1)=\frac{1}{\sqrt{N}}$.

3. For $-2^{J-2} \leq k \leq 2^{J-2}-1$ and $1 \leq j \leq J-1$, define $\hat{\varphi}\left(2^{j}(2 k+1)\right)$ according to $(3.5)$, i.e.,

$$
\hat{\varphi}\left(2^{j}(2 k+1)\right)=m_{0}\left(2^{j-1}(2 k+1)\right) \hat{\varphi}\left(2^{j-1}(2 k+1)\right) .
$$

The remainder of the proof will justify this definition. Observe that because $m_{0}(0)=1,(3.3)$ implies $m_{0}\left(2^{J-1}\right)=0$, which forces each dyadic sequence to terminate after $2^{J-1}$ steps. Indeed,

$$
\hat{\varphi}\left(2^{J}(2 k+1)\right)=\hat{\varphi}\left(2^{J+1} k+2^{J}\right)=m_{0}\left(2^{J-1}\right) \hat{\varphi}\left(2^{J} k+2^{J-1}\right)=0,
$$

which shows both that the above definition satisfies (3.5) for all $k \in \mathbb{Z}$ and that the corresponding refinable function, $\varphi$, is a trigonometric polynomial of degree at most $2^{J-1}\left(2^{-1} N-1\right)$.

It must now be shown that (3.6) holds for each $n \in \mathbb{Z}_{N}$. The $n=0$ case rests solely on $\hat{\varphi}(0)$, which is immediate based on the construction above. Moreover, the recursion (3.5) implies that for each odd $n \in \mathbb{Z}_{N}$, only the coefficients $\hat{\varphi}(n)$ and $\hat{\varphi}(n-N)$ contribute to (3.6) and only one of these is nonzero. Thus, the construction above also guarantees (3.6) is satisfied for each odd $n \in \mathbb{Z}_{N}$.

It remains to demonstrate (3.6) for nonzero, even $n \in \mathbb{Z}_{N}$, a set of $2^{J-1}-1$ elements. In order to determine the coset in which a given Fourier coefficient will occur, the map $k \mapsto 2 k$ must be understood on $\mathbb{Z}_{N}$. The doubling map on $\mathbb{Z}_{N}$ is two-to-one and satisfies

$$
2^{j}(2 k+1)=2^{j}\left(2 k+1+2^{-j} m N\right), \quad-2^{j-1} \leq m \leq 2^{j-1}-1,
$$

for $j \geq 0$. Consider the $j=1$ version of this identity, which states that $2(2 k+1)$ and $2(2 k+1-N / 2)$ are equivalent in $\mathbb{Z}_{N}$. Suppose that $n=2(2 k+1)$, where $0<2 k+1<2^{-1} N$. It follows from the above construction that 
$2 k+1$ and $2 k+1-N / 2$ are the only indices corresponding to nonzero Fourier coefficients of $\hat{\varphi}$ whose double is congruent to $n$. In other words,

$$
\sum_{k \in \mathbb{Z}}|\hat{\varphi}(n+k N)|^{2}=\frac{1}{N}\left(\left|m_{0}(2 k+1)\right|^{2}+\left|m_{0}(2 k+1-N / 2)\right|^{2}\right)=\frac{1}{N} .
$$

Considering the range $1 \leq 2 k+1 \leq 2^{-1} N-1$, observe that $n$ takes on $2^{J-2}$ distinct values in $\mathbb{Z}_{N}$.

In general, for $1 \leq j \leq J-1,2^{J-j-1}$ distinct indices $n$ occur in (3.7) over the range $1 \leq 2 k+1 \leq 2^{-1} N$, each with multiplicity $2^{j+1}$. The multiplicity is the maximum possible after doubling $j$ times, so the corresponding indices must be distinct both among themselves and from those for smaller values of $j$. Notice that as $j$ ranges from 1 to $J-2$ the number of distinct values is given by $2^{J-2}+2^{J-3}+\cdots+1=2^{J-1}-1$, precisely the number of indices unaccounted for above. Hence, the validity of the construction rests on sums of the form,

$$
\sum_{m=-2^{j-1}}^{2^{j-1}-1}\left|\hat{\varphi}\left(2^{j}\left(2 k+1+2^{-j} m N\right)\right)\right|^{2}, \quad 0 \leq k \leq \frac{N}{4}-1 .
$$

Employing (3.5) and (3.3) while noting that all of the nonzero, odd Fourier coefficients of $\hat{\varphi}$ are identical, it follows that

$$
\begin{aligned}
\sum_{m=-2^{j-1}}^{2^{j-1}-1}\left|\hat{\varphi}\left(2^{j}\left(2 k+1+2^{-j} m N\right)\right)\right|^{2} & \sum_{m=-2^{j-1}} \prod_{\ell=0}^{2^{j-1}-1}\left|m_{0}\left(2^{\ell}\left(2 k+1+2^{-j} m N\right)\right)\right|^{2}\left|\hat{\varphi}\left(2 k+1+2^{-j} m N\right)\right|^{2} \\
= & \frac{1}{N} \sum_{m=-2^{j-2}}^{2^{j-2}-1} \prod_{\ell=1}^{j-1}\left|m_{0}\left(2^{\ell}\left(2 k+1+2^{-j} m N\right)\right)\right|^{2} \\
& \times\left(\left|m_{0}\left(2 k+1+2^{-j} m N\right)\right|^{2}+\left|m_{0}\left(2 k+1+2^{-j} m N-2^{-1} N\right)\right|^{2}\right) \\
= & \frac{1}{N} \sum_{m=-2^{j-2}}^{2^{j-2}-1} \prod_{\ell=1}^{j-1}\left|m_{0}\left(2^{\ell}\left(2 k+1+2^{-(j-1)} m N\right)\right)\right|^{2} .
\end{aligned}
$$

By extracting the lowest-index term from the product at each successive step in the computation, this eventually reduces to a single summand $\frac{1}{N}$, completing the proof.

The construction described in the proof of Theorem 3.6 is just one possibility among infinitely many choices. However, the chosen construction has the advantage of leading to a scaling function of minimal degree. The next proposition shows that the low-pass filter associated with an orthonormal 
scaling function on the real line often induces a scaling function for an MRA on the circle through Theorem 3.6.

Proposition 3.7. Suppose $c \in \ell^{2}(\mathbb{Z})$ is an absolutely summable sequence whose Fourier transform $m=\hat{c}$ satisfies

$$
|m(\xi)|^{2}+\left|m\left(\xi+2^{-1}\right)\right|^{2}=1, \quad \xi \in \mathbb{T},
$$

If $c_{0} \in \ell\left(\mathbb{Z}_{N}\right)$ is defined by

$$
c_{0}(n)=\sum_{k \in \mathbb{Z}} c(n+k N), \quad n \in \mathbb{Z}_{N}
$$

where $N=2^{J}(J \in \mathbb{N})$, then $m_{0}=\sqrt{N} \widehat{c_{0}}$ satisfies (3.3).

Proof. The absolute summability of $c$ ensures both that the definition of $c_{0}$ makes sense and that pointwise evaluation of $m$ is appropriate. Notice that

$$
\begin{aligned}
m_{0}(n) & =\sum_{k \in \mathbb{Z}_{N}} \sum_{\ell \in \mathbb{Z}} c(k+\ell N) e^{-2 \pi i n(k+\ell N) / N} \\
& =\sum_{k \in \mathbb{Z}} c(k) e^{-2 \pi i n k / N}=m(n / N),
\end{aligned}
$$

while $m_{0}\left(n+2^{-1} N\right)=m\left(n / N+2^{-1}\right)$ by a similar calculation. Thus,

$$
\left|m_{0}(n)\right|^{2}+\left|m_{0}(n+N / 2)\right|^{2}=|m(n / N)|^{2}+\left|m\left(n / N+2^{-1}\right)\right|^{2}=1, \quad n \in \mathbb{Z}_{N},
$$

proving that (3.3) holds.

ExAMPLE 3.8. It will be beneficial to discuss a concrete example of the construction described in the proof of Theorem 3.6. Fix $N=8$ and consider the low-pass filter induced by the Haar mask $c \in \ell\left(\mathbb{Z}_{N}\right)$ given by $c(0)=$ $c(1)=\frac{1}{2}$ with $c(n)=0$ for $n \neq 0,1$. The low-pass filter $m_{0} \in \ell\left(\mathbb{Z}_{N}\right)$ is given by

$$
m_{0}(n)=e^{-\pi i n / 8} \cos (n \pi / 8), \quad n \in \mathbb{Z}_{8} .
$$

This filter satisfies (3.3) as a consequence of Proposition 3.7. The construction used to prove Theorem 3.6 leads to the definition of $\varphi$ by

$$
\begin{aligned}
\hat{\varphi}(-3) & =\frac{1}{\sqrt{8}} \longrightarrow \hat{\varphi}(-6)=\hat{\varphi}(-3) m_{0}(5) \longrightarrow \hat{\varphi}(-12)=\hat{\varphi}(-6) m_{0}(5) m_{0}(2), \\
\hat{\varphi}(-1) & =\frac{1}{\sqrt{8}} \longrightarrow \hat{\varphi}(-2)=\hat{\varphi}(-1) m_{0}(7) \longrightarrow \hat{\varphi}(-4)=\hat{\varphi}(-2) m_{0}(7) m_{0}(6), \\
\hat{\varphi}(0) & =\frac{1}{\sqrt{8}} \\
\hat{\varphi}(1) & =\frac{1}{\sqrt{8}} \longrightarrow \hat{\varphi}(2)=\hat{\varphi}(1) m_{0}(1) \longrightarrow \hat{\varphi}(4)=\hat{\varphi}(1) m_{0}(1) m_{0}(2), \\
\hat{\varphi}(3) & =\frac{1}{\sqrt{8}} \longrightarrow \hat{\varphi}(6)=\hat{\varphi}(3) m_{0}(3) \longrightarrow \hat{\varphi}(12)=\hat{\varphi}(6) m_{0}(3) m_{0}(6),
\end{aligned}
$$


where each strand terminates because $m_{0}(4)=0$. This definition makes $\varphi$ refinable of order 8 and it is evident that $\varphi$ is a real-valued trigonometric polynomial of degree 12 . It is routine to verify that $[\hat{\varphi}, \hat{\varphi}]_{N}(n)=1$ for $n \in \mathbb{Z}_{8}$, showing that $\varphi$ is an orthonormal scaling function. Figure 1 shows the graph of $\varphi$ on $\mathbb{T}$.

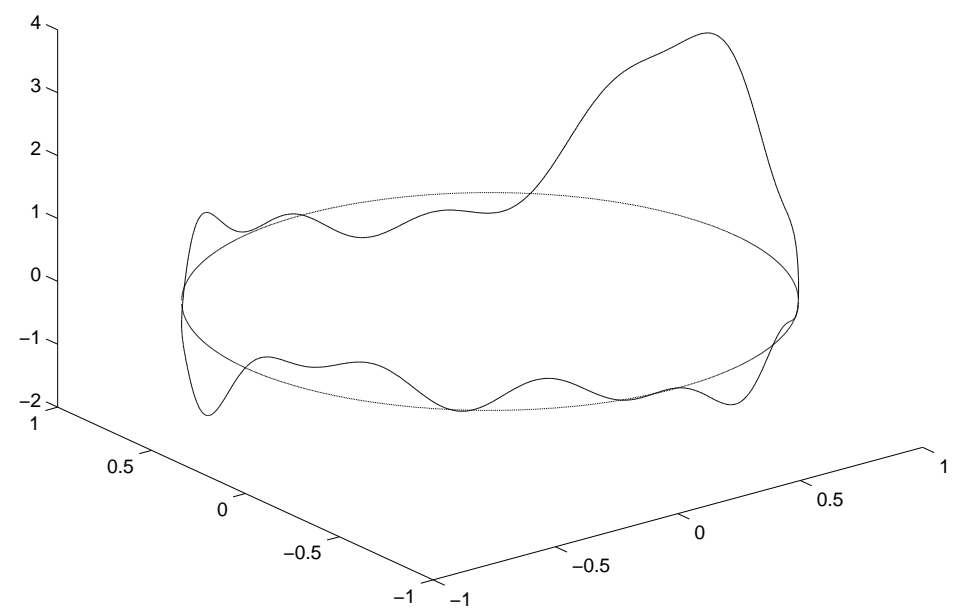

FIGURE 1. Haar scaling function $\varphi$ of order $N=8$.

Remark 3.9. Consider the stretched Haar filter,

$$
m(\xi)=\frac{1}{2}\left(1+e^{-2 \pi i 3 \xi}\right),
$$

which fails to produce an orthonormal scaling function on the line. Proposition 3.7 shows how this filter can be used to produce finite-dimensional filters satisfying $m_{0}(0)=1$ and the Smith-Barnwell identity, (3.3). Consequently, one can also construct corresponding orthonormal scaling functions in light of Theorem 3.6.

\section{MRA WAVELEtS}

Given an MRA of the sort described in the previous section, one hopes to obtain an orthonormal wavelet basis for $V_{0} \ominus V_{J}$. This is the motivation for the following definition. 
Definition 4.1. Let $\left\{V_{j}\right\}_{j=0}^{J}$ be an $M R A$ of order $N=2^{J}(J \in \mathbb{N})$. A function $\psi \in V_{0}$ is a wavelet for the MRA if the collection

$$
\left\{2^{\frac{j}{2}} T_{2^{-j} N}^{n} D^{j-1} \psi: 1 \leq j \leq J, n \in \mathbb{Z}_{2^{-j} N}\right\}
$$

is an orthonormal basis for $V_{0} \ominus V_{J}$.

The construction of an MRA wavelet will center on a decomposition of $V_{j}$ as $V_{j}=V_{j+1} \oplus W_{j+1}, 0 \leq j \leq J-1$, where $W_{j}$ is of the form $W_{j+1}=$ $V_{2^{-(j+1)} N}\left(D^{j} \psi\right)$. The main result of this section shows that the decomposition just described is possible through a natural extension of the notion of a highpass filter on the line $([5,7])$.

THEOREM 4.2. Suppose that $\varphi$ is the scaling function of an MRA of order $N=2^{J} \quad(J \in \mathbb{N})$ and define $\psi \in V_{0}$ by

$$
\hat{\psi}(k)=m_{1}(k) \hat{\varphi}(k), \quad k \in \mathbb{Z},
$$

where $m_{1} \in \ell\left(\mathbb{Z}_{N}\right)$ is chosen as

$$
m_{1}(n)=\overline{m_{0}\left(n+2^{-1} N\right)} e^{-2 \pi i n / N}, \quad n \in \mathbb{Z}_{N} .
$$

Then $\psi$ is a wavelet for the MRA.

Proof. First it will be shown that $W_{1}$ is orthogonal to $V_{1}$ via the bracket product,

$$
\begin{aligned}
{\left[2^{\frac{1}{2}} \widehat{D \varphi}, 2^{\frac{1}{2}} \hat{\psi}\right]_{\frac{N}{2}}(n)=} & N \sum_{k \in \mathbb{Z}} \widehat{D \varphi}(n+k N / 2) \overline{\hat{\psi}(n+k N / 2)} \\
= & N \sum_{k \in \mathbb{Z}}|\hat{\varphi}(n+k N / 2)|^{2} m_{0}(n+k N / 2) \overline{m_{1}(n+k N / 2)} \\
= & N\left[\sum_{k \in \mathbb{Z}}|\hat{\varphi}(n+k N)|^{2} m_{0}(n) \overline{m_{1}(n)}\right. \\
& \left.+\sum_{k \in \mathbb{Z}}|\hat{\varphi}(n+N / 2+k N)|^{2} m_{0}(n+N / 2) \overline{m_{1}(n+N / 2)}\right] \\
= & m_{0}(n) \overline{m_{1}(n)}+m_{0}(n+N / 2) \overline{m_{1}(n+N / 2)} \\
= & 0,
\end{aligned}
$$

using (3.3) and (4.1). The fact that $W_{1}$ is the orthogonal complement of $V_{1}$ in $V_{0}$ follows from dimensional considerations, while the orthonormality of 
$X_{2^{-1} N}\left(2^{\frac{1}{2}} \psi\right)$ follows from the calculation,

$$
\begin{aligned}
{\left[2^{\frac{1}{2}} \hat{\psi}, 2^{\frac{1}{2}} \hat{\psi}\right]_{2^{-1} N}(n)=} & N \sum_{k \in \mathbb{Z}}|\hat{\psi}(n+k N / 2)|^{2} \\
= & N \sum_{k \in \mathbb{Z}}|\hat{\varphi}(n+k N / 2)|^{2}\left|m_{1}(n+k N / 2)\right|^{2} \\
= & N\left[\sum_{k \in \mathbb{Z}}|\hat{\varphi}(n+k N)|^{2}\left|m_{1}(n)\right|^{2}\right. \\
& \left.+\sum_{k \in \mathbb{Z}}|\hat{\varphi}(n+N / 2+k N)|^{2}\left|m_{1}(n+N / 2)\right|^{2}\right] \\
= & \left|m_{0}(k+N / 2)\right|^{2}+\left|m_{0}(k+N / 2)\right|^{2} \\
= & 1 .
\end{aligned}
$$

The picture at scale $j$ is analogous, in light of Remark 3.4, so the result follows by induction on $j$ as in Theorem 3.5.

EXAMPLE 4.3. Returning to the Haar example of the previous section, let $m_{1} \in \ell\left(\mathbb{Z}_{8}\right)$ chosen according to (4.1) with $m_{0}$ as in Example 3.8. This leads to a trigonometric polynomial orthonormal wavelet $\psi$ of degree 12 , which is depicted in Figure 2.

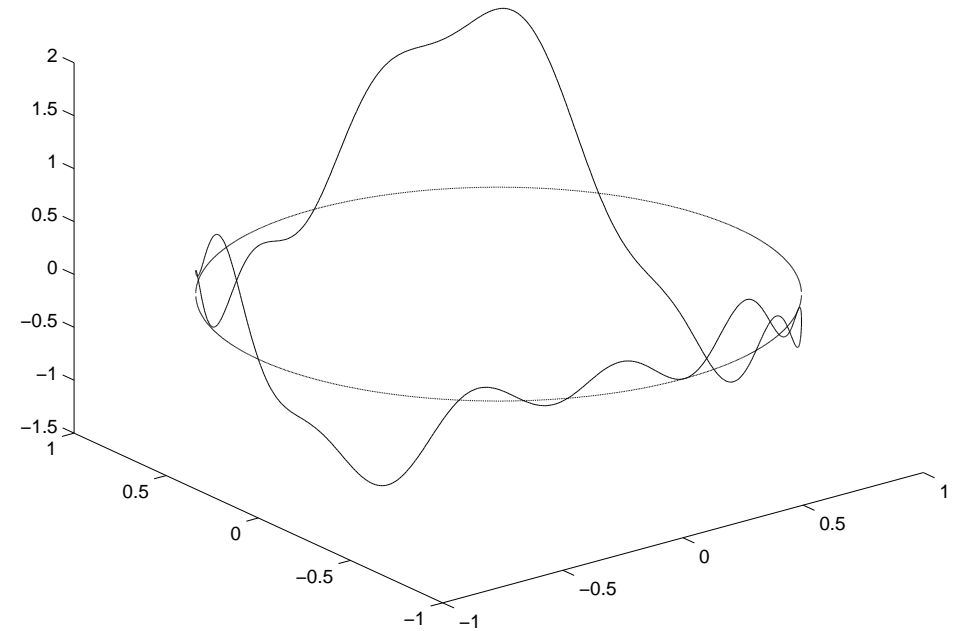

Figure 2. Haar wavelet $\psi$ of order $N=8$. 


\section{Approximation}

The goal of this section is to show that MRAs of finite order can be used to obtain arbitrarily close approximations of functions in $L^{2}(\mathbb{T})$.

Lemma 5.1. Suppose that $\varphi$ is a scaling function for an MRA of order $N=2^{J}(J \in \mathbb{N})$. The orthogonal projection onto $V_{0}=V_{N}(\varphi)$ is described by

$$
\widehat{P f}(k)=[\hat{f}, \hat{\varphi}]_{N}(k) \hat{\varphi}(k), \quad k \in \mathbb{Z} .
$$

ProOf. Because $X_{N}(\varphi)$ forms an orthonormal basis for $V_{N}(\varphi)$ the orthogonal projection is given by

$$
P f=\sum_{n \in \mathbb{Z}_{N}}\left\langle f, T^{n} \varphi\right\rangle T^{n} \varphi .
$$

Under the Fourier transform this is equivalent to

$$
\widehat{\operatorname{Pf}}(k)=\sum_{n \in \mathbb{Z}_{N}}\left\langle f, T^{n} \varphi\right\rangle e^{-2 \pi i n k / N} \hat{\varphi}(k),
$$

which reduces to the claimed expression as a consequence of Proposition 2.2.

Suppose that $f$ is a trigonometric monomial, i.e., $\hat{f}(k)=\delta_{r k}$ for some $r \in \mathbb{Z}$. In this case, $[\hat{f}, \hat{\varphi}]_{N}$ is given by

$$
[\hat{f}, \hat{\varphi}]_{N}(n)= \begin{cases}0, & n \not \equiv r \\ N \overline{\hat{\varphi}(r)}, & n \equiv r .\end{cases}
$$

The error of approximation is thus given by

$$
\|P f-f\|^{2}=\left(N|\hat{\varphi}(r)|^{2}-1\right)^{2}+\sum_{k \neq 0}|N \hat{\varphi}(r) \hat{\varphi}(r+k N)|^{2},
$$

but the fact that $X_{N}(\varphi)$ is an orthonormal basis means

$$
1=[\hat{\varphi}, \hat{\varphi}]_{N}(r)=N|\hat{\varphi}(r)|^{2}+N \sum_{k \neq 0}|\hat{\varphi}(r+k N)|^{2} .
$$

Hence, the approximation error can be rewritten as

$$
\|P f-f\|^{2}=\left(N|\hat{\varphi}(r)|^{2}-1\right)^{2}+N|\hat{\varphi}(r)|^{2}\left(1-N|\hat{\varphi}(r)|^{2}\right)=1-N|\hat{\varphi}(r)|^{2},
$$

which tends to zero as $|\hat{\varphi}(r)| \rightarrow \frac{1}{\sqrt{N}}$. Recall that the construction used to prove Theorem 3.6 begins with $\hat{\varphi}(k)=\frac{1}{\sqrt{N}}$ for all odd $k$ satisfying $|k|<$ $\frac{N}{2}$. Therefore, these scaling functions lead to perfect approximation of the corresponding odd trigonometric monomials. The approximation error for even trigonometric monomials rests on the properties of the low-pass filter $m_{0}$.

Assume that $m(\xi)$ is a continuous function on the circle satisfying $m(0)=$ 1 and (3.3). Given $N=2^{J}(J \in \mathbb{N}), m$ induces a low-pass filter $m_{0} \in \ell\left(\mathbb{Z}_{N}\right)$ 
according to Proposition 3.7. One can then construct a scaling function $\varphi$ associated to $m_{0}$ through the construction described in the proof of Theorem 3.6. Define the error of approximation, $E_{N}(k)$ by

$$
E_{N}(k)=\left(1-N|\hat{\varphi}(k)|^{2}\right)^{\frac{1}{2}}, \quad k \in \mathbb{Z} .
$$

Hence, $E_{N}(k)$ represents the approximation error $\|P f-f\|$ where $f=e^{2 \pi i k x}$ and $P$ is the orthogonal projection onto $V_{N}(\varphi)$. The following proposition shows that arbitrarily small approximation error can be achieved using an MRA of sufficiently high order.

Proposition 5.2. Fix $r \in \mathbb{N}$ and $\varepsilon>0$. Then there exists $N=2^{J}$ $(J \in \mathbb{N})$ such that $E_{N}(k)<\varepsilon$ for $|k|<r$, where $\varphi$ is constructed as above.

Proof. Let $j=\left\lceil\log _{2} r\right\rceil$, then each integer $|k|<r$ can be written as $k=2^{\ell} n$ where $0 \leq \ell \leq j$ and $n<r$ is odd. Because $m$ is continuous and $m(0)=1$, there exists $\delta>0$ such that

$$
\left|m_{0}(\xi)\right|^{j}>\sqrt{1-\varepsilon^{2}}, \quad|\xi|<\delta .
$$

Choose $J \in \mathbb{N}$ such that $\frac{r}{N}=2^{-J} r<\delta$. Thus, if $|k|<r$ and $k=2^{\ell} n$ (as above), then

$$
|\hat{\varphi}(k)|=\frac{1}{\sqrt{N}} \prod_{p=0}^{\ell-1}\left|m_{0}\left(2^{p} n\right)\right|=\frac{1}{\sqrt{N}} \prod_{p=0}^{\ell-1}\left|m\left(2^{p} n / N\right)\right|>\frac{1}{\sqrt{N}} \sqrt{1-\varepsilon^{2}}
$$

and, consequently, $E_{N}(k)<\varepsilon$, as desired.

ExAmPle 5.3. Proposition 5.2 certainly does not represent the only path to good approximation with the finite MRAs considered here. Motivated by the Shannon wavelet on the line, let $m_{0} \in \ell\left(\mathbb{Z}_{N}\right)$ be defined by

$$
m_{0}(n)= \begin{cases}1, & n<\frac{N}{4} \text { or } n>\frac{3 N}{4}, \\ \frac{1}{\sqrt{2}}, & n=\frac{N}{4} \text { or } n=\frac{3 N}{4}, \quad n \in \mathbb{Z}_{N}, \\ 0, & \text { otherwise }\end{cases}
$$

where $N=2^{J}$ for a natural number $J>2$. Because $m_{0}$ satisfies (3.3) with $m_{0}(0)=1$ there is an orthonormal scaling function $\varphi$ associated to $m_{0}$. In particular, if $\varphi$ is constructed as in Theorem 3.6 it follows that $\hat{\varphi}(k)=\frac{1}{\sqrt{N}}$ whenever $|k|<\frac{N}{2}$. Thus, the associated error of approximation $E_{N}(k)$ is zero for $|k|<\frac{N}{2}$. The scaling function for this example corresponding to $N=64$ is shown below as Figure 3. The corresponding wavelet with $N=64$ is shown in Figure 4. 


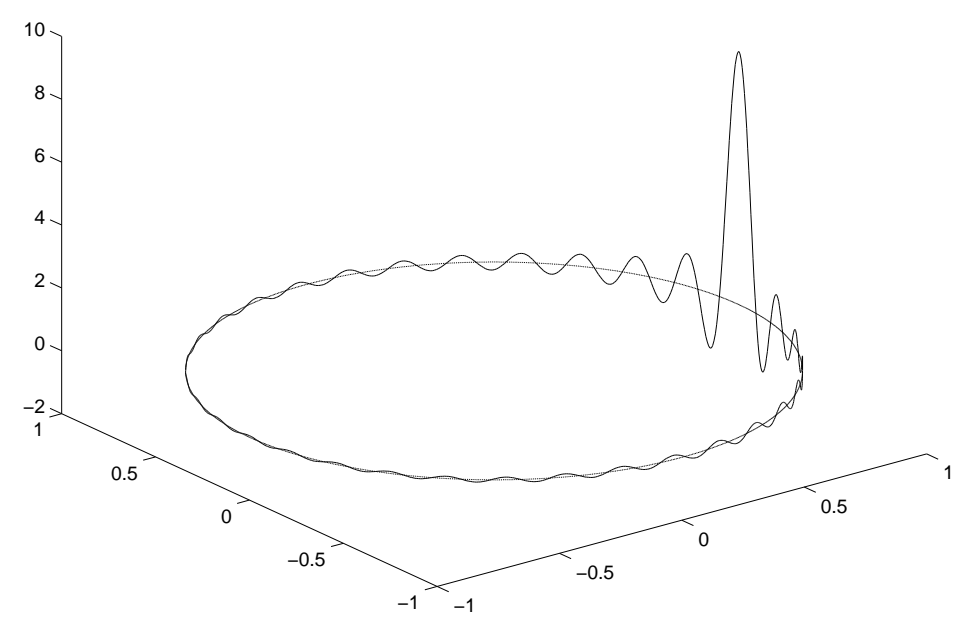

Figure 3. Shannon-like scaling function $\phi$ of order $N=64$.

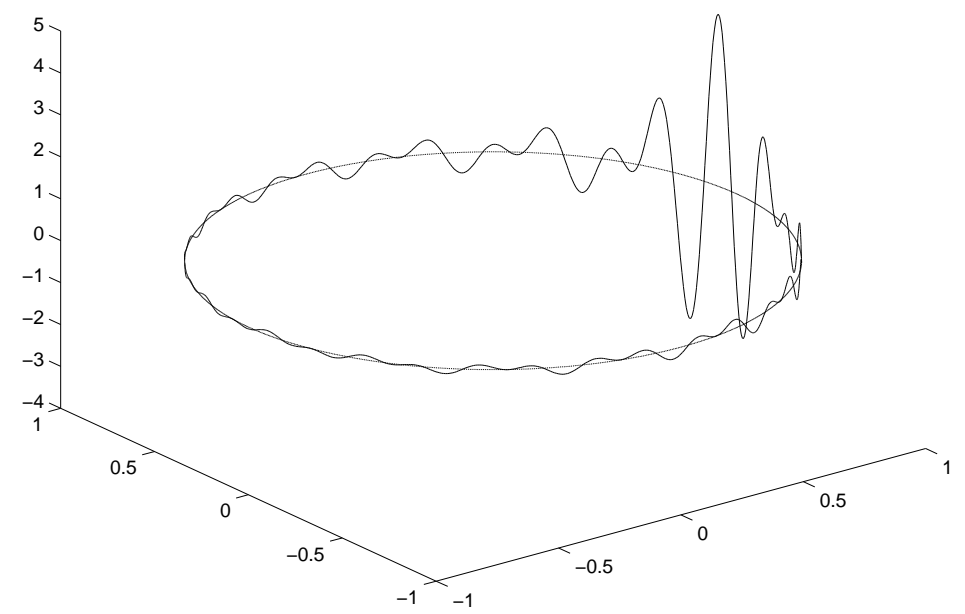

Figure 4. Shannon-like wavelet $\psi$ of order $N=64$. 


\section{REFERENCES}

[1] C. de Boor, R. A. DeVore and A. Ron, The structure of finitely generated shiftinvariant spaces in $L^{2}\left(\mathbb{R}^{d}\right)$, J. Funct. Anal. 119 (1994), 37-78.

[2] G. Walter and L. Cai, Periodic wavelets from scratch, J. Comput. Anal. Appl. 1 (1999), $25-41$.

[3] C. K. Chui and H. N. Mhaskar, On trigonometric wavelets, Constr. Approx. 9 (1993), $167-190$.

[4] O. Christensen, Frames and bases. An introductory course, Birkhaüser Boston, Inc. Boston, 2008

[5] I. Daubechies, Ten lectures on wavelets, CBMS-NSF Regional Conference Series in Applied Mathematics 61, SIAM, Philadelphia, 1992

[6] P. Gressman, Wavelets on the integers, Collect. Math. 52 (2001), 257-288.

[7] E. Hernández and G. Weiss, A first course on wavelets, CRC Press, Boca Raton, 1996.

[8] Y. Meyer, Wavelets and operators, Cambridge University Press, Cambridge, 1992.

[9] G. Plonka and M. Tasche, A unified approach to periodic wavelets, "Wavelets: theory, algorithms, and applications", Academic Press, San Diego, 1994, 137-151.

[10] A. Ron and Z. Shen, Frames and stable bases for shift-invariant subspaces of $L^{2}\left(\mathbb{R}^{d}\right)$, Canad. J. Math. 47 (1995), 1051-1094.

B. D. Johnson

Department of Mathematics and Computer Science

Saint Louis University

St. Louis, MO 63103

USA

Received: 6.3.2010. 